\title{
Analysis of the finite difference time domain technique to solve the Schrödinger equation for quantum devices
}

\author{
Antonio Soriano ${ }^{\text {a) }}$ \\ Department of Applied Physics, University of Granada. Granada 18071. Spain \\ Enrique A. Navarro ${ }^{\text {b) }}$ \\ Department of Applied Physics, University of Valencia. Burjasot 46100. Spain \\ Jorge A. Portíc) \\ Department of Applied Physics, University of Granada. Granada 18071. Spain \\ Vicente Such ${ }^{\text {d) }}$ \\ Department of Applied Physics, University of Valencia. Burjasot 46100. Spain
}

(Received 11 November 2003; accepted 1 April 2004)

\begin{abstract}
An extension of the finite difference time domain is applied to solve the Schrödinger equation. A systematic analysis of stability and convergence of this technique is carried out in this article. The numerical scheme used to solve the Schrödinger equation differs from the scheme found in electromagnetics. Also, the unit cell employed to model quantum devices is different from the Yee cell used by the electrical engineering community. A bound for the time step is derived to ensure stability. Several numerical experiments in quantum structures demonstrate the accuracy of a second order, comparable to the analysis of electromagnetic devices with the Yee cell. (C) 2004 American Institute of Physics. [DOI: 10.1063/1.1753661]
\end{abstract}

\section{INTRODUCTION}

The finite difference in time domain (FDTD) is a widely used tool in electromagnetics. The application of FDTD technique for the analysis of quantum devices (FDTD-Q) is based on the FDTD for electromagnetics, and extends this technique to solve the Schrödinger equation. The FDTD method solves the Maxwell curl equations using discrete derivative operators. ${ }^{1}$ In the same way, the FDTD-Q solves a discretized Schrödinger equation in an iterative process.

The FDTD-Q technique has been successfully used to solve the problem of quantum dots (QD) in Refs. 2 and 3. However, some aspects of the FDTD-Q cell, convergency, and stability of the numerical scheme have not been explored until now. In this article, the FDTD-Q technique is revised and analyzed when applied to the Schrödinger equation with a space-dependent potential, this is the case of QD or quantum wires $(\mathrm{QW})$.

In one-dimensional cases, the numerical solution of the Schrödinger equation is usually based on matrix methods like Numerov's technique. ${ }^{4}$ In cylindrical and spherical geometry it is possible to reduce the dimensionality by considering the symmetry of the problem, and rewriting it using a suitable coordinate system. The computational cost increases rapidly with matrix techniques when is not possible to reduce the problem to the one-dimensional case. A solution to reduce the computational cost in two- and three-dimensional problems is to use finite differences algorithms. The FDTD

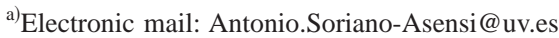

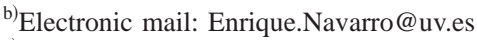

${ }^{c}$ Electronic mail: jporti@ugr.es

d)Electronic mail: Vicente.Such@uv.es
}

technique has been successfully applied in electromagnetics, and we have now extended this method to solve the Schrödinger equation. The FDTD-Q will provide us with a very useful tool for the numerical study of quantum devices, like QD and QW, which require a two- or three-dimensional coordinate system to be correctly modeled.

The FDTD-Q technique simulates the time evolution of the wave function in a three-dimensional space, where the potential could be any arbitrary function. We will show in this article how numerical values of the wave function in the time marching scheme of FDTD-Q represent a good approach to the time behavior of the wave function for the modeled structure.

In this article we present a detailed formulation of the numerical FDTD-Q technique for the general threedimensional Schrödinger equation. We analyze the stability of the time marching FDTD-Q scheme and derive a bound for the time step. The maximum time step is a bound that avoids the accumulation of numerical error. It is a must for the stability of the time domain simulation in the same way as the Courant stability condition in the FDTD technique for electromagnetics. Finally, calculations with the FDTD-Q have been carried out simulating a quantum device, specifically a quantum well wire (QWW) with a known analytical solution. There are many numerical experiments, using different mesh resolutions, with different cell sizes, which analyze the numerical convergence of the method. The numerical error is calculated by comparing the numerical solutions with the known analytical wave functions. Some plots of the error versus cell size show that a second order accuracy is achieved in the numerically obtained FDTD-Q wave function. 


\section{FINITE DIFFERENCE TIME DOMAIN TECHNIQUE FOR QUANTUM DEVICES}

A formal description of the FDTD-Q technique is introduced in this section. We explain step by step how to deduce the discrete equations, and how to use them to obtain the numerical solution of the Schrödinger equation. First, the complex wave function is separated into two real functions that correspond to its real and imaginary parts

$$
\Psi(\vec{r}, t)=\Psi_{R}(\vec{r}, t)+j \Psi_{I}(\vec{r}, t) .
$$

Then, the Schrödinger equation

$$
j \hbar \frac{\partial \Psi(\vec{r}, t)}{\partial t}=-\frac{\hbar^{2}}{2 m_{e}} \vec{\nabla}^{2} \Psi(\vec{r}, t)+V(\vec{r}) \Psi(\vec{r}, t)
$$

is divided into two equations involving real functions corresponding to $\Psi_{R}$ and $\Psi_{I}$

$$
\begin{aligned}
\hbar \frac{\partial \Psi_{R}(\vec{r}, t)}{\partial t}= & -\frac{\hbar^{2}}{2 m_{e}}\left[\frac{\partial^{2} \Psi_{I}(\vec{r}, t)}{\partial x^{2}}+\frac{\partial^{2} \Psi_{I}(\vec{r}, t)}{\partial y^{2}}\right. \\
& \left.+\frac{\partial^{2} \Psi_{I}(\vec{r}, t)}{\partial z^{2}}\right]+V(\vec{r}) \Psi_{I}(\vec{r}, t), \\
\hbar \frac{\partial \Psi_{I}(\vec{r}, t)}{\partial t}= & \frac{\hbar^{2}}{2 m_{e}}\left[\frac{\partial^{2} \Psi_{R}(\vec{r}, t)}{\partial x^{2}}+\frac{\partial^{2} \Psi_{R}(\vec{r}, t)}{\partial y^{2}}\right. \\
& \left.+\frac{\partial^{2} \Psi_{R}(\vec{r}, t)}{\partial z^{2}}\right]-V(\vec{r}) \Psi_{R}(\vec{r}, t) .
\end{aligned}
$$

A mesh is defined in a given boundary value problem, where the continuous complex wave function is represented in our computational domain as two discrete functions, the discretized real part of the wave function and the discretized imaginary part of the wave function

$$
\begin{aligned}
& \Psi_{R}(\vec{r}, t) \simeq \Psi_{R}^{n}(i, j, k)=\Psi_{R}(i \Delta x, j \Delta y, k \Delta z, n \Delta t), \\
& \Psi_{I}(\vec{r}, t) \simeq \Psi_{I}^{n}(i, j, k)=\Psi_{I}(i \Delta x, j \Delta y, k \Delta z, n \Delta t) .
\end{aligned}
$$

The second-order derivatives in Eqs. (3a) and (3b) are discretized using centered differences. Therefore, the calculation of Eqs. (3a) and (3b) at a given mesh point $(i, j, k)$ involves the points $(i+1, j, k),(i-1, j, k),(i, j+1, k),(i, j$ $-1, k),(i, j, k+1)$, and $(i, j, k-1) .{ }^{1}$ For example, to update the real part of the wave function at the discrete point $(i, j, k)$; at the instant $(n+1) \Delta t$, we need the imaginary part of the wave function at the points $(i, j, k),(i+1, j, k),(i$ $-1, j, k), \quad(i, j+1, k), \quad(i, j-1, k), \quad(i, j, k+1), \quad$ and $(i, j, k$ $-1)$ at the instant $(n+1 / 2) \Delta t$. This evaluation involves the real and imaginary parts of the wave function at each node. Second-order derivatives are calculated at each point of the discrete space then, in our numerical scheme both the real and imaginary parts of the wave function are located at the same nodes. This detail makes a difference with the FDTD technique when applied to electromagnetic problems. The unit cell in FDTD-Q is shown in Fig. 1.

Once the differential operators are replaced by their corresponding discrete operators using centered differences, we

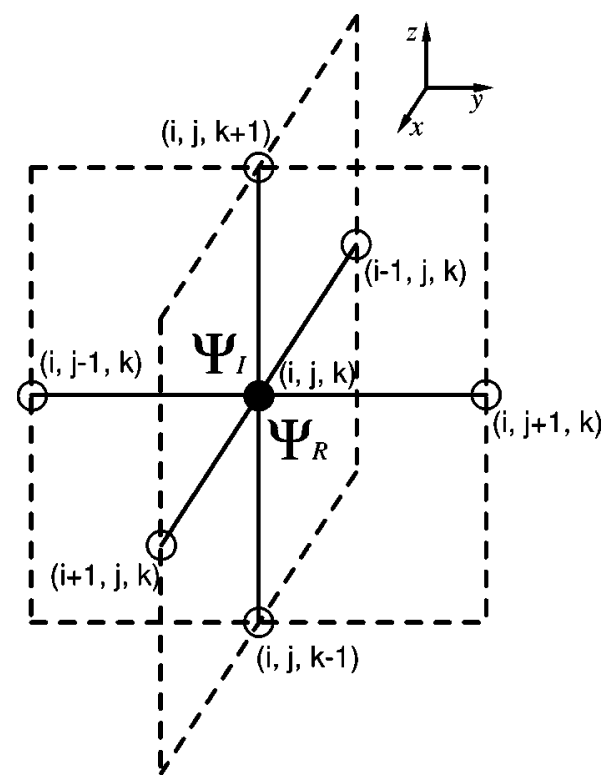

FIG. 1. FDTD-Q cell.

write $\Psi_{R}^{n+1}$ as a function of $\Psi_{R}^{n}$ and $\Psi_{I}^{n+1 / 2}$. The FDTD-Q equation obtained to update the real part of the wave function is

$$
\begin{aligned}
\Psi_{R}^{n+1}(i, j, k)= & \Psi_{R}^{n}(i, j, k)+\left[\frac{\hbar \Delta t}{m_{e}}\left(\frac{1}{\Delta x^{2}}+\frac{1}{\Delta y^{2}}+\frac{1}{\Delta z^{2}}\right)\right. \\
& \left.+\frac{V(i, j, k) \Delta t}{\hbar}\right] \Psi_{i}^{n+1 / 2}(i, j, k)-\frac{\hbar \Delta t}{2 m_{e} \Delta x^{2}} \\
& \times\left(\Psi_{i}^{n+1 / 2}(i+1, j, k)+\Psi_{i}^{n+1 / 2}(i-1, j, k)\right) \\
& -\frac{\hbar \Delta t}{2 m_{e} \Delta y^{2}}\left(\Psi_{i}^{n+1 / 2}(i, j+1, k)\right. \\
& \left.+\Psi_{i}^{n+1 / 2}(i, j-1, k)\right)-\frac{\hbar \Delta t}{2 m_{e} \Delta z^{2}} \\
& \times\left(\Psi_{i}^{n+1 / 2}(i, j, k+1)+\Psi_{i}^{n+1 / 2}(i, j, k-1)\right)
\end{aligned}
$$

with a similar equation for the imaginary part.

The above equations are solved in an iterative process, with the necessary boundary conditions, in which the time is incremented $\Delta t$ in the numerical loop.

Numerical FDTD-Q loop:

(1) Impose initial boundary conditions on $\Psi_{R}$ and $\Psi_{I}$ at $t=0$.

(2) Calculation of $\Psi_{I}$ at time $t+\Delta t / 2$.

(3) Boundary conditions on $\Psi_{I}$.

(4) Calculation of $\Psi_{R}$ at time $t+\Delta t$, using Eq. (5).

(5) Boundary conditions on $\Psi_{R}$.

(6) Time is incremented $t=t+\Delta t$.

(7) If $t<N \Delta t$ then Goto 2. Else STOP the loop.

The wave function propagates in the numerical space of the computer following the above iterative process. In this way, it simulates the time behavior of the wave function, 
derived from the time domain Schrödinger equation. The loop stops when the condition $t=N \Delta t$ is verified, $N \Delta t$ is the total simulation time.

A rough analogy between the FDTD technique in electromagnetism and the above presented FDTD-Q is the identification of the electric field components with the real part of the wave function, and the magnetic field components with the imaginary part. However, some epistemological differences arise. The FDTD technique was developed to solve the Maxwell curl equations involving the electromagnetic fields, that are vectorial magnitudes; whereas Schrödinger equation is a scalar equation involving the scalar wave function. Differences can also be extended to the boundary conditions: The boundary conditions in FDTD are defined in the field components separately, up to six electromagnetic components. Whereas in FDTD-Q the boundary conditions are enforced only on $\Psi_{R}$ and $\Psi_{I}$. Also, the electric and magnetic field components are sampled at distinct spatial points, whereas the real and imaginary parts of the wave function are evaluated at the same spatial point.

The computational cost of FDTD-Q is lower than the cost of the FDTD technique used for electromagnetic problems. There are two components per cell in FDTD-Q, real and imaginary parts of the wave function, and six field components per cell in FDTD, three electric and three magnetic.

\section{STABILITY}

The time discretization $\Delta t$ is the time increment between consecutively calculated fields in the numerical approach described in Eq. (5). The choice of $\Delta t$ is critical in the FDTD-Q simulations. The computational cost decreases as $\Delta t$ increases, because longer time steps provide us with longer simulation times with the same number of numerical iterations. On the other hand, from our numerical experiments, the longer the time steps are the less stable is the behavior of our simulation.

The time step should be chosen as a balance between computational cost and stability. Of course, the best time step to choose would be the longer one so that the algorithm's stability is maintained. So, it is important to establish a maximum time step that ensures the stability of our simulation. A relationship between the spatial and temporal discretizations is necessary to keep the numerical error under control as the simulation process progresses.

If we separate the finite difference problem into two eigenvalue problems, a spatial eigenvalue and a temporal eigenvalue problem, the study of the numerical stability is analytically feasible. The wave packet is supposed to be a superposition of plane waves, and each plane wave is an eigenfunction of the numerical domain. The eigenvalues associated to the spatial differentiation and the eigenvalues associated to the temporal operators are calculated. If the spatial eigenvalue spectrum is enclosed in the temporal eigenvalue spectrum then the algorithm will be numerically stable. ${ }^{5}$ In this way, the numerical stability is guaranteed by preventing the uncontrolled growth of the numerical error at each time iteration.

\section{A. Temporal eigenvalues}

The temporal eigenvalues $\lambda$ of the Schrödinger equation are analyzed from left side of Eq. (2)

$$
j \hbar \frac{\partial \Psi}{\partial t}=\lambda \Psi .
$$

Where both the real and imaginary parts of $\Psi$ are considered, the differential operator is introduced, through its equivalent discrete operator, to obtain a numerical differentiation:

$$
j \hbar \frac{\Psi^{n+1 / 2}(i, j, k)-\Psi^{n-1 / 2}(i, j, k)}{\Delta t}=\lambda \Psi^{n}(i, j, k) .
$$

The "growth factor" $q$ determines the growth of the wave function at each time iteration

$$
q=\frac{\Psi^{n+1 / 2}}{\Psi^{n}}
$$

if $q$ is introduced in Eq. (7), and the resulting equation is solved in order to obtain the growth factor as a function of $\Delta t$ and $\lambda$

$$
\begin{aligned}
& q^{2}+j \frac{\lambda \Delta t}{\hbar} q-1=0, \\
& q=-j \frac{\lambda \Delta t}{2 \hbar} \pm \sqrt{1-\left(\frac{\lambda \Delta t}{2 \hbar}\right)^{2}} .
\end{aligned}
$$

The instability appears when the eigenfunction associated to the $\lambda$ eigenvalue grows on each iteration. The stability of the temporal dependence of any spatial mode is assumed by imposing the condition $|q| \leqslant 1$. This is verified automatically if

- $\operatorname{Im}(\lambda)=0$ : This condition establishes that all eigenvalues must be real. If we assume that the Hamiltonian is an hermitian operator, it will always be true because all eigenvalues associated to an hermitian operator are real.

- $\operatorname{Re}(\lambda) \Delta t \leqslant 2 \hbar$ : A stability condition is derived from this relationship, involving both the spatial and temporal discretization.

\section{B. Spatial eigenvalues}

The process described above is repeated again for the spatial eigenvalues. The right side of Eq. (2) is analyzed as a spatial eigenvalue problem

$$
\begin{aligned}
\lambda_{s} \Psi(\vec{r}, t)= & -\frac{\hbar^{2}}{2 m}\left(\frac{\partial^{2} \Psi(\vec{r}, t)}{\partial x^{2}}+\frac{\partial^{2} \Psi(\vec{r}, t)}{\partial y^{2}}+\frac{\partial^{2} \Psi(\vec{r}, t)}{\partial z^{2}}\right) \\
& +V(\vec{r}) \Psi(\vec{r}, t) .
\end{aligned}
$$

The most general solution for the wave equation can be described as a superposition of plane waves.

$$
\Psi(x, y, z, t)=e^{j\left(k_{x} x+k_{y} y+k_{z} z-\omega t\right)} .
$$

The operator $\nabla^{2}$ is replaced by another expression that is the resulting from its application to a plane wave $\Psi(x, y, z, t) \approx e^{j\left(k_{x} i \Delta x+k_{y} j \Delta y+k_{z} k \Delta z-\omega n \Delta t\right)}$ 


$$
\begin{aligned}
\frac{\partial^{2} \Psi(x, y, z, t)}{\partial x^{2}} & \approx \frac{e^{j\left(k_{x}(i+1) \Delta x\right)}-2 e^{j\left(k_{x} i \Delta x\right)}+e^{j\left(k_{x}(i-1) \Delta x\right)}}{\Delta x^{2}} \\
& =-4 \sin ^{2}\left(\frac{k_{x} \Delta x}{2}\right) \Psi^{n}(i, j, k)
\end{aligned}
$$

The associated eigenvalues are derived

$$
\begin{aligned}
\lambda_{s}= & \frac{2 \hbar^{2}}{m}\left[\frac{\sin ^{2}\left(\frac{k_{x} \Delta x}{2}\right)}{\Delta x^{2}}+\frac{\sin ^{2}\left(\frac{k_{y} \Delta y}{2}\right)}{\Delta y^{2}}+\frac{\sin ^{2}\left(\frac{k_{z} \Delta z}{2}\right)}{\Delta z^{2}}\right] \\
& +V .
\end{aligned}
$$

The numerical solution requires that both the temporal and spatial eigenvalues associated to the temporal and spatial parts of the Schrödinger equation are the same. Taking into account the conditions imposed to get $|q| \leqslant 1$ the following relationship is obtained:

$$
\begin{aligned}
\frac{2 \hbar}{\Delta t} & \geqslant \frac{2 \hbar^{2}}{m}\left[\frac{\sin ^{2}\left(\frac{k_{x} \Delta x}{2}\right)}{\Delta x^{2}}+\frac{\sin ^{2}\left(\frac{k_{y} \Delta y}{2}\right)}{\Delta y^{2}}+\frac{\sin ^{2}\left(\frac{k_{z} \Delta z}{2}\right)}{\Delta z^{2}}\right]+V \\
& \leqslant \frac{2 \hbar^{2}}{m}\left[\frac{1}{\Delta x^{2}}+\frac{1}{\Delta y^{2}}+\frac{1}{\Delta z^{2}}\right]+V \\
& \geqslant \frac{\hbar^{2}}{m}\left[\frac{1}{\Delta x^{2}}+\frac{1}{\Delta y^{2}}+\frac{1}{\Delta z^{2}}\right]+V .
\end{aligned}
$$

Writing $\Delta t$ as a function of the spatial discretization and remaining constants, a maximum value for the time step that ensures the stability of the algorithm can be obtained:

$$
\Delta t \leqslant \frac{\hbar}{\frac{\hbar^{2}}{m}\left[\frac{1}{\Delta x^{2}}+\frac{1}{\Delta y^{2}}+\frac{1}{\Delta z^{2}}\right]+V}
$$

We named "critical step" or $\Delta t_{\text {critical }}$, the maximum time step which maintains the algorithm's stability, this value is obtained from the right side of Eq. (16).

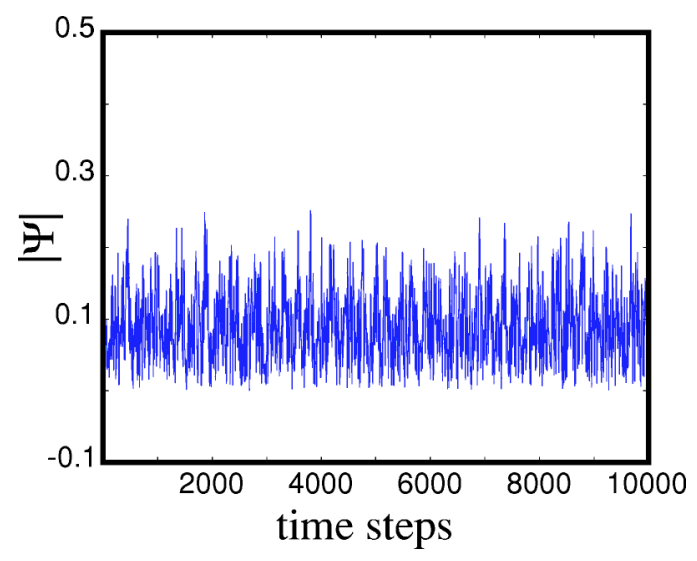

FIG. 2. Absolute value of the wave function at an arbitrary point of the mesh during the first ten thousand iterations. $\Delta t=\Delta t_{\text {critical }}$.

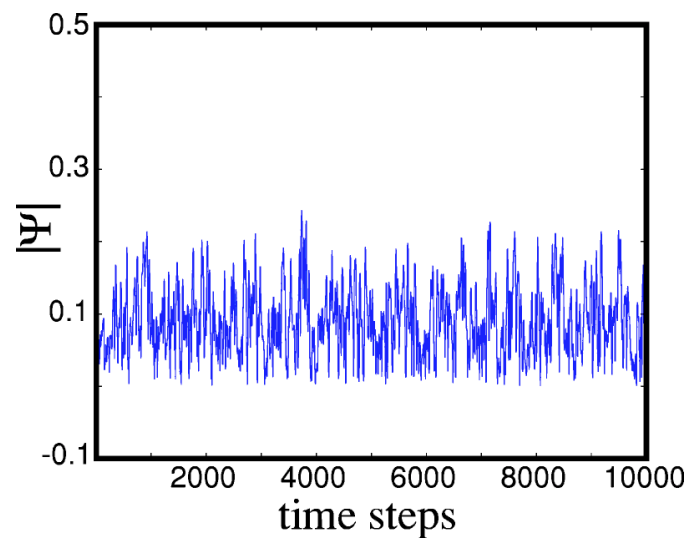

FIG. 3. Absolute value of the wave function at an arbitrary point of the mesh during the first ten thousand iterations. $\Delta t=0.5 \Delta t_{\text {critical }}$.

Some numerical experiments were carried out to check the validity of the above stability condition. In all cases, the stability was observed when the condition of Eq. (16) was verified.

A rectangular two-dimensional QWW was simulated with a regular mesh $\Delta x=\Delta y$. The simulation time was $N \Delta t$, where the number of time iterations was $N \geqslant 100000$, and $\Delta t$ was varied around $\Delta t_{\text {critical }}$.

The excitation consists of a set of narrow pulses in the time domain that are arbitrarily distributed on the cross section of the QWW. The boundary conditions in our example were introduced in the wave function by defining a rectangular boundary in which the potential is infinite. Then, the wave function is zero at that boundary.

The time evolution of the wave function at a given point of the mesh was filed at each step of the simulation loop. The time evolution of the wave function is plotted in Figs. 2, 3, and 4 for three distinct $\Delta t$. The wave function evolved under a bound when the time step was lower than $\Delta t_{\text {critical }}$. In Figs. 2 and 3 we show two different simulations for the first ten thousand iterations of the FDTD-Q simulation. However, during more than one million time steps of the simulations, not shown in Figs. 2 and 3, the maximum value of the wave
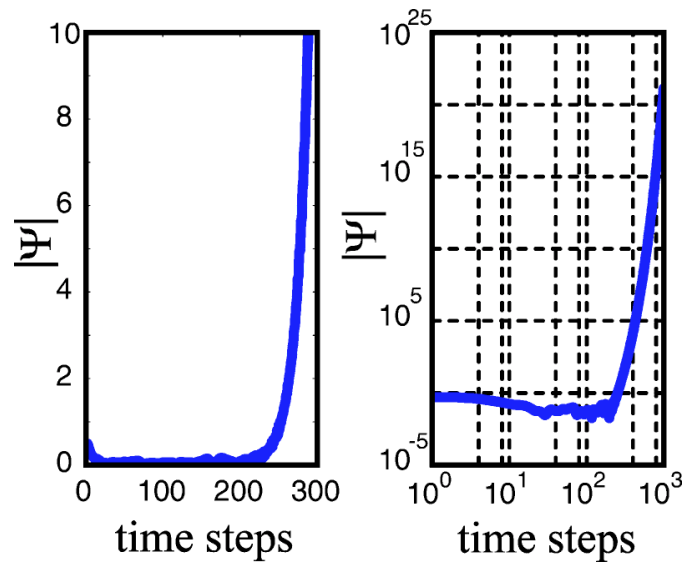

FIG. 4. Time evolution of the wave function at an arbitrary point of the mesh. $\Delta t=1.005 \Delta t_{\text {critical }}$. Left: First three hundred iterations. Right: The first one thousand iterations. 
function can be observed evolving under a bound. That is, stability was observed when $\Delta t \leqslant \Delta t_{\text {critical }}$.

Numerical experiments with a time step longer than $\Delta t_{\text {critical }}$ are observed to diverge. In Fig. 4 the time evolution of the wave function at a given point of the mesh for $\Delta t$ $=1.005 \Delta t_{\text {critical }}$ is presented. In the logarithmic scale of Fig. 4 we can see how the FDTD-Q algorithm diverges quickly when the selected time step is a little greater than $\Delta t_{\text {critical }}$.

\section{CONVERGENCE}

Once the stability of the FDTD-Q algorithm is ensured, under certain conditions about $\Delta t$, the next step consists of checking that the numerical solution obtained with FDTD-Q converges in a given boundary value problem. The study of the convergence has been carried out by analyzing a quantum structure with known analytical solution, this is the case of the QWW that are numerically treated as two-dimensional structures. Then, a large set of numerical simulations have been carried out for a given QW, and FDTD-Q results are compared against analytically obtained solutions. A QWW was simulated with different spatial discretizations, in order to analyze the numerical error as a function of the spatial discretization. The errors were calculated as the difference between the numerical and analytically obtained eigenvalues and eigenfunctions.

The analysis of a particle in a QWW is mathematically similar to the analysis of an electromagnetic waveguide. ${ }^{6}$

\section{A. Numerical eigenvalues and eigenfunctions in a QWW}

After the introduction of a time-domain-space-domain pulsed excitation, only eigenfunctions are allowed inside the two-dimensional cross-section of the QWW. These have time harmonic dependence with the energies of the bound states. After the time pulsed excitation the wave function can be expressed as a superposition of eigenfunctions, whose eigenvalues are included in the spectrum of the introduced time domain pulse

$$
\Psi(\vec{r}, t)=\sum_{m=1}^{\infty} A_{m} \varphi_{m}(\vec{r}) e^{-j E_{m} t / h} .
$$

In the above equation $\varphi_{m}(\vec{r})$ appears, it is the space distribution of the $m$-eigenfunction along the cross section of the QWW, and the eigenvalue or eigenenergy $E_{m}$ associated to the $m$-eigenfunction. The complex constant $A_{m}$ establishes the contribution of the $m$-eigenfunction to the wave function.

Equations (3a) and (3b) are defined for a given spatial discretization $\Delta t$ chosen along with boundary conditions defining the edges of the QWW. The potential is assumed to become infinite at the walls of the QWW, so the wave function becomes zero there. From the FDTD-Q algorithm, the real and imaginary parts of the wave function are computed in alternate time steps, giving the time evolution of the wave function components $\Psi_{R}$ and $\Psi_{I}$.

From now on we will use a two-dimensional description of the FDTD-Q equations, because the QWW constitutes a two-dimensional structure with translational symmetry along the $z$-axis. In particular, the FDTD-Q technique provides a time series at a given mesh point $(i, j)$

$$
\Psi^{n}(i, j)=\Psi(i \Delta x, j \Delta y, n \Delta t) \quad n=0,1,2, \ldots, N-1,
$$

where the total simulation time is $N \Delta t$.

A method to reconstruct the eigenfunctions is described in Ref. 2, in the discrete case it is the evaluation of the equation

$$
\varphi_{m}(i, j)=\frac{1}{\sqrt{N}} \sum_{n=0}^{N-1} \Psi^{n}(i, j) e^{-j E_{m} n \Delta t / \hbar} .
$$

The application of the inverse discrete fourier transform (IDFT) to $\Psi^{n}(i, j)$ in Eq. (19) will result in a discrete function of frequency,

$$
\begin{aligned}
\varphi_{m}(i, j) & =\frac{1}{N} \sum_{n=0}^{N-1}\left(\sum_{p=0}^{N-1} \psi_{p}(i, j) e^{j(2 \pi p / N) n}\right) e^{-j E_{m} n \Delta t / \hbar} \\
& =\frac{1}{N} \sum_{p=0}^{N-1} \psi_{p}(i, j) \frac{1-e^{-j\left(E_{m} N \Delta t\right) / \hbar}}{1-e^{-j\left(\left(E_{m} \Delta t\right) / \hbar-(2 \pi p / N)\right.}} .
\end{aligned}
$$

This equation shows that the information to reconstruct each eigenfunction is shared in all discrete frequency components. If we pay attention to the term [1 $\left.-e^{-j\left(\left(E_{m} \Delta t\right) / \hbar-(2 \pi p) / N\right)}\right]$, we notice that the most important contribution to the $m$-eigenfunction is due to the component named $p_{m}$ which makes the following term the closest to zero:

$$
\left(\frac{E_{m} \Delta t}{\hbar}-\frac{2 \pi p_{m}}{N}\right) \approx 0
$$

Numerically, the eigenvalue associated to the $m$-eigenfunction is evaluated as

$$
E_{m-\mathrm{FDTD}}=\frac{2 \pi \hbar}{N \Delta t} p_{m}
$$

and the energy sensitivity achievable by the discrete fourier transform (DFT) is

$$
\Delta E=\frac{2 \pi \hbar}{N \Delta t},
$$

which is related to the number of time steps $N$, and the cell dimensions $\Delta x$ and $\Delta y$ by the stability criterion derived above. Apart from this factor, the achievable accuracy to be gained in the numerical calculation also depends on the space-time discretization process undertaken by the FDTD-Q. This will be discussed in the following section.

The numerical error also depends on the feasibility of the imposed numerical boundary conditions, and inevitable numerical roundoff. The relative error between numerical and analytical energies is calculated to evaluate the accuracy of our procedure

$$
e_{r}(\%)=\frac{\left|E_{m-\mathrm{FDTD}}-E_{m \text {-analytical }}\right|}{\left|E_{m \text {-analytical }}\right|} 100 .
$$

The field distribution for each eigenfunction can be calculated once the eigenvalue is known. The shape of each 
TABLE I. Estimated FDTD-Q energy levels for the $224 \times 336 \mathrm{~nm}^{2}$ quantum well wire in million electron volt, and their relative error.

\begin{tabular}{|c|c|c|c|c|c|c|c|c|}
\hline \multirow[b]{2}{*}{ mesh } & \multicolumn{2}{|c|}{ Eigenfunction 1-1 } & \multicolumn{2}{|c|}{ Eigenfunction 2-2 } & \multicolumn{2}{|c|}{ Eigenfunction 1-4 } & \multicolumn{2}{|c|}{ Eigenfunction 3-2 } \\
\hline & energy (meV) & $\%$ & energy (meV) & $\%$ & energy (meV) & $\%$ & energy (meV) & $\%$ \\
\hline $6 \times 9$ & $1.068710^{-2}$ & 1.27 & $4.030510^{-2}$ & 6.92 & $5.313010^{-2}$ & 12.6 & $6.870210^{-2}$ & 14.9 \\
\hline $10 \times 15$ & $1.068710^{-2}$ & 1.27 & $4.213710^{-2}$ & 2.69 & $5.771010^{-2}$ & 5.06 & $7.603010^{-2}$ & 5.871 \\
\hline $12 \times 18$ & $1.068710^{-2}$ & 1.27 & $4.244310^{-2}$ & 1.98 & $5.862610^{-2}$ & 3.55 & $7.725210^{-2}$ & 4.36 \\
\hline $14 \times 21$ & $1.068710^{-2}$ & 1.27 & $4.274810^{-2}$ & 1.27 & $5.923710^{-2}$ & 2.55 & $7.816810^{-2}$ & 3.22 \\
\hline $16 \times 24$ & $1.068710^{-2}$ & 1.27 & $4.274810^{-2}$ & 1.27 & $5.954210^{-2}$ & 2.05 & $7.877810^{-2}$ & 2.47 \\
\hline $18 \times 27$ & $1.068710^{-2}$ & 1.27 & $4.305310^{-2}$ & 0.57 & $5.984710^{-2}$ & 1.55 & $7.908410^{-2}$ & 2.09 \\
\hline $20 \times 30$ & $1.068710^{-2}$ & 1.27 & $4.305310^{-2}$ & 0.57 & $6.015310^{-2}$ & 1.04 & $7.938910^{-2}$ & 1.71 \\
\hline $22 \times 33$ & $1.068710^{-2}$ & 1.27 & $4.305310^{-2}$ & 0.57 & $6.015310^{-2}$ & 1.04 & $7.969410^{-2}$ & 1.33 \\
\hline $24 \times 36$ & $1.068710^{-2}$ & 1.27 & $4.305310^{-2}$ & 0.57 & $6.015310^{-2}$ & 1.04 & $8.000010^{-2}$ & 0.96 \\
\hline $28 \times 42$ & $1.068710^{-2}$ & 1.27 & $4.305310^{-2}$ & 0.57 & $6.045810^{-2}$ & 0.54 & $8.000010^{-2}$ & 0.96 \\
\hline $32 \times 48$ & $1.068710^{-2}$ & 1.27 & $4.305310^{-2}$ & 0.57 & $6.045810^{-2}$ & 0.54 & $8.030510^{-2}$ & 0.58 \\
\hline $36 \times 54$ & $1.068710^{-2}$ & 1.27 & $4.335910^{-2}$ & 0.14 & $6.045810^{-2}$ & 0.54 & $8.030510^{-2}$ & 0.58 \\
\hline $40 \times 60$ & $1.068710^{-2}$ & 1.27 & $4.335910^{-2}$ & 0.14 & $6.045810^{-2}$ & 0.54 & $8.030510^{-2}$ & 0.58 \\
\hline $44 \times 66$ & $1.068710^{-2}$ & 1.27 & $4.335910^{-2}$ & 0.14 & $6.076310^{-2}$ & 0.04 & $8.061110^{-2}$ & 0.20 \\
\hline
\end{tabular}

$m$-eigenfunction is obtained by calculating the DFT component associated to the energy $E_{m}$, over the entire cross section of the QWW.

\section{B. Numerical analysis of eigenvalues}

In order to simplify the numerical analysis we simulated a two-dimensional QWW. To avoid degeneration along $x$ and $y$ axis, we simulated a $\mathrm{QWW}$ with a rectangular cross section of $224 \times 336 \mathrm{~nm}^{2}$.

After the introduction of the time-domain-space-domain pulsed excitation in the two-dimensional FDTD-Q mesh, the wave function is sampled at different mesh points, providing the time domain numerical behavior of the wave function. The sampling was done at three different points of the mesh to avoid nulls of the eigenfunctions. The DFT of these temporal series provide us with the spectral amplitude of the wave function in the sampled mesh points. This frequency response has maximum values, or peaks, at the frequencies associated to the eigenenergies of the confined states. These are the $E_{m \text {-FDTD }}$ numerical values.

The accuracy in the numerical estimation of the obtained eigenvalues is expected to depend on the spatial discretization. As cell dimensions decrease, numerical eigenvalues become closer to the analytical values given in the following equation:

$$
E_{p, q}=\frac{\pi^{2} \hbar^{2}}{2 m_{e}}\left(\frac{p^{2}}{a^{2}}+\frac{q^{2}}{b^{2}}\right),
$$

where $a=224 \mathrm{~nm}$ and $b=336 \mathrm{~nm}$ are the dimensions of the QWW, the integers $p$ and $q$ define each eigenvalue.

As we have already said, $\Delta t$ is limited by cell dimensions to ensure the algorithm's stability. The number of time iterations $N$ was increased for smaller cell dimensions, in order to maintain the DFT sensitivity when the same QWW is simulated with denser meshes. The error's decrease in the eigenvalue estimation when the mesh size increases is mainly due to the effect of cell dimensions, because the DFT resolution remains constant.

Table I shows the FDTD-Q calculated eigenvalues, and their deviation from the analytical ones. The eigenenergies of
$E_{1-1}, E_{2-2}, E_{1-4}$, and $E_{3-2}$ are compared in Table I to show the convergence of our technique. In Table II we show the convergence of the energy levels when the sensitivity of the DFT is increased. The increase of $N$ leads to greater accuracy in the estimation of the eigenvalues, see Eq. (23). However there is no linear relationship between the deviation and numerical results in Table II. The deviation in the numerical energy levels is associated to the discretization, and we obtain these numerical results using the DFT, however the DFT sensitivity covers the numerical error. Then, by increasing the DFT sensitivity we observe a convergence of the deviation to a constant value that is associated to the numerical discretization $40 \times 60$ cells.

\section{Numerical analysis of eigenfunctions}

The numerically obtained eigenfunction comes closer to the analytical value when cell dimensions are reduced. The most general solution of the Schrödinger equation for a par-

TABLE II. Estimated FDTD-Q energy levels for the $224 \times 336 \mathrm{~nm}^{2}$ quantum well wire in million electron volt, and their relative error. The mesh size is $40 \times 60$ cells, $\Delta t=1.354410^{-17} \mathrm{~s}$.

\begin{tabular}{rcccc}
\hline \hline & \multicolumn{2}{c}{ Eigenfunction 1-1 } & \multicolumn{2}{c}{ Eigenfunction 2-2 } \\
\cline { 2 - 5 } Iterations & Energy (meV) & Error (\%) & Energy (meV) & Error (\%) \\
\hline 5000 & $1.221410^{-2}$ & 12.829 & $4.274810^{-2}$ & 1.275 \\
8000 & $1.145010^{-2}$ & 5.777 & $4.198510^{-2}$ & 3.038 \\
9000 & $1.017810^{-2}$ & 5.976 & $4.410510^{-2}$ & 1.859 \\
10000 & $1.221410^{-2}$ & 12.829 & $4.274810^{-2}$ & 1.275 \\
20000 & $1.068710^{-2}$ & 1.275 & $4.274810^{-2}$ & 1.275 \\
30000 & $1.119610^{-2}$ & 3.426 & $4.274810^{-2}$ & 1.275 \\
50000 & $1.099210^{-2}$ & 1.546 & $4.335910^{-2}$ & 0.135 \\
80000 & $1.068710^{-2}$ & 1.275 & $4.313010^{-2}$ & 0.394 \\
90000 & $1.085710^{-2}$ & 0.292 & $4.308710^{-2}$ & 0.491 \\
100000 & $1.068710^{-2}$ & 1.275 & $4.335910^{-2}$ & 0.135 \\
200000 & $1.084010^{-2}$ & 0.135 & $4.320610^{-2}$ & 0.217 \\
300000 & $1.078910^{-2}$ & 0.335 & $4.325710^{-2}$ & 0.100 \\
500000 & $1.080910^{-2}$ & 0.147 & $4.323710^{-2}$ & 0.147 \\
800000 & $1.080210^{-2}$ & 0.217 & $4.324410^{-2}$ & 0.129 \\
900000 & $1.082310^{-2}$ & 0.021 & $4.322310^{-2}$ & 0.178 \\
1000000 & $1.080910^{-2}$ & 0.147 & $4.323710^{-2}$ & 0.147 \\
\hline \hline
\end{tabular}



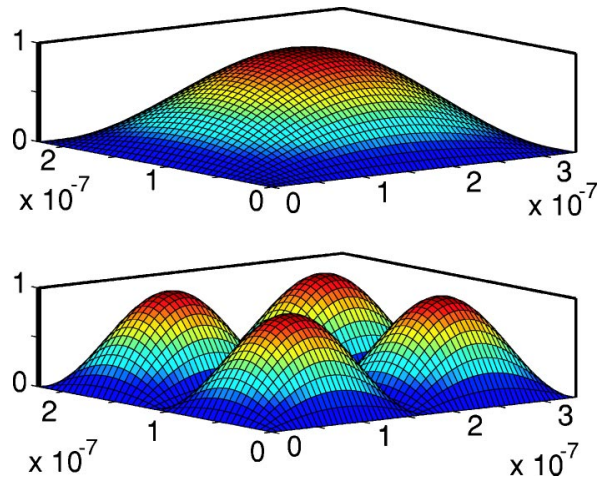

FIG. 5. Numerical eigenfunctions of the $224 \times 336 \mathrm{~nm}^{2}$ two-dimensional quantum well, regular mesh of $40 \times 60$ square cells. Up: eigenfunction 1-1. Down: eigenfunction 2-2.

ticle in a rectangular QWW can be written as a linear combination of its corresponding eigenfunctions, see Eq. (26). In a two dimensional case

$$
\begin{aligned}
& \Psi(x, y, t)=\sum_{p=1}^{\infty} \sum_{q=1}^{\infty} A_{p, q} \varphi_{p, q}(x, y) e^{-j\left(E_{p, q}\right) t / \hbar}, \\
& \varphi_{p, q}(x, y)=\sin \left(\frac{\pi p}{a} x\right) \sin \left(\frac{\pi q}{b} y\right),
\end{aligned}
$$

where $\varphi_{p, q}(x, y)$ is the space distribution of the $(p, q)$-eigenfunction along the cross section of the QWW, its associated eigenenergy is $E_{p, q}$, and $A_{p, q}$ is a complex constant that establishes the contribution of the $(p, q)$ eigenfunction to the wave function.

To obtain the eigenfunctions, ${ }^{6}$ the DFT is performed during the FDTD-Q simulation at each mesh point [see Eq. (19)]. The DFT is done at some selected frequencies associated to the lowest numerical eigenenergies obtained in the eigenvalue analysis of previous section. Figure 5 shows the calculated FDTD-Q eigenfunctions (1-1) and (2-2).

To analyze the convergence of the FDTD-Q, we evaluated the deviation, or error, as the difference between analytical and numerical solutions at each mesh point $(i, j) .{ }^{7} \mathrm{In}$ this particular case, maximum absolute error and average error were obtained for two given eigenfunctions: The 1-1, and 2-2. The average or mean error is calculated averaging the error at all the mesh points for a given mesh. In Fig. 6 we present the maximum and average error for the first numerical eigenfunction (1-1) versus cell size. The least-squares regression provides a slope of 1.74 for the average error, and 1.51 for the maximum error, in both cases the correlation coefficient is higher than 0.9. Similar results for the 2-2 eigenfunction are presented in Fig. 7, where the least-squares regression gives a slope of 2.35 for the average error and 2.11 for the maximum error, also with correlation coefficients higher than 0.9 . The regression line of the error versus cell size is also plotted in Figs. 6 and 7 for both the average and maximum error, where the convergence versus cell size of the proposed technique is demonstrated. The average error is more significant and reduces faster (versus cell size) than

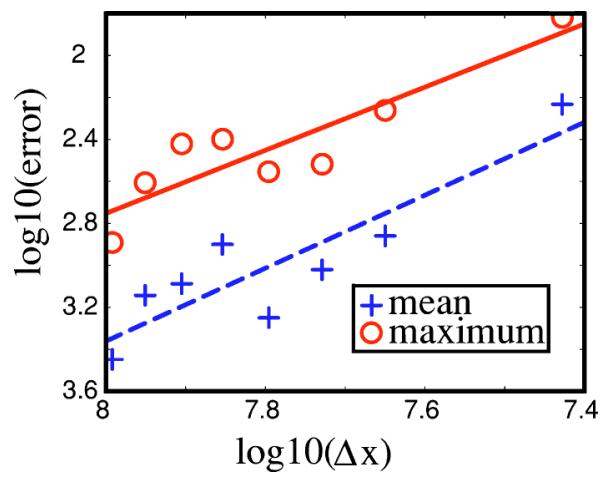

FIG. 6. Decimal logarithm of the numerical error in calculating the 1-1 eigenfunction versus decimal logarithm of the cell size: $(\bigcirc)$ maximum error, and $(+)$ average error.

the maximum error. Then, we can say, as a first approach, that the calculation of the eigenfunctions is second-order accurate versus discretization.

A second-order accuracy was expected in the finite difference time domain results, because centered differences approach for numerical derivatives provide a second-order accuracy. ${ }^{1}$ However, the used excitation and the numerical DFT influenced the final error for the numerically obtained eigenfunctions in the spectral domain. We observed this influence in our simulations. The pulsed excitation distributes its energy between the eigenfunctions, but the best coupling between excitation and a given eigenfunction is when the pulse is located in the maximum of the spectral distribution. The location of the maximum is not known beforehand, then heuristic excitation can distort the spectral distribution of the numerically calculated eigenfunction. The DFT also influences the accuracy of the numerically obtained eigenfunctions, because its sensitivity influences the numerical spectrum.

\section{CONCLUSIONS}

The numerical finite difference time domain technique that solves the Schrödinger equation is named FDTD-Q. In the present work, the FDTD-Q is presented, analyzed, and successfully applied in the analysis of a cross-sectional QWW. A relationship between the spatial discretization and

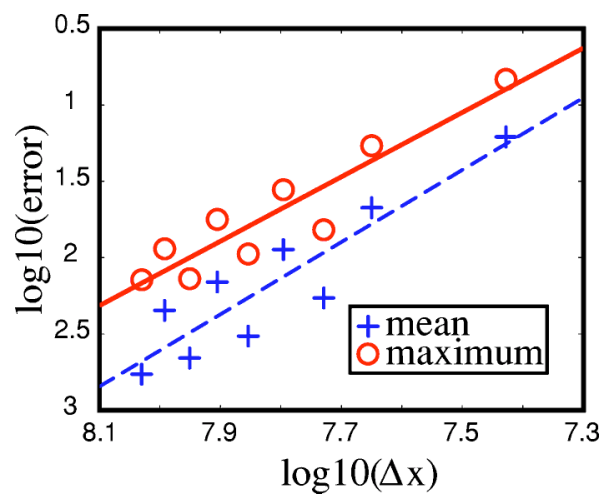

FIG. 7. Decimal logarithm of the numerical error in calculating the 2-2 eigenfunction versus decimal logarithm of the cell size: $(\bigcirc)$ maximum error, and $(+)$ average error. 
the maximum time increment is derived to ensure the stability of the numerical technique. A detailed analysis of the convergence is carried out, with many numerical experiments simulating a QWW. The FDTD-Q has a unit cell distinct from the Yee cell, however a second-order dependence is obtained in the analysis of the numerical error versus the discretization. Our results validate the use of the FDTD-Q in the analysis of quantum devices like QWW and QW, and guarantee a future successful application in threedimensional structures like QD.

\section{ACKNOWLEDGMENTS}

This work has been partially supported by the Spanish Ministerio de Ciencia y Tecnologia, Project No. TIC-2002-
4096-C03-01. Also, it is supported by the Spanish Ministerio de Educacion, Cultura y Deportes, Grant No. AP2001-3741.

${ }^{1}$ J. Nouguier, Metodos de Calcul Numerique, Paris, 1983.

${ }^{2}$ D. Sullivan and D. Citrin, J. Appl. Phys. 91, 3219 (2002).

${ }^{3}$ D. Sullivan and D. Citrin, J. Appl. Phys. 89, 3841 (2001).

${ }^{4} \mathrm{~F}$. Vesely, Computational Physics-An Introduction, 2nd ed. (Plenum, New York, 1994).

${ }^{5}$ E. A. Navarro, PhD Thesis, Universidad de Valencia, 1992.

${ }^{6}$ E. A. Navarro and V. Such, IEE Proc., Part H: Microwaves, Antennas Propag. 139, 491 (1992).

${ }^{7}$ E. A. Navarro, N. T. Sangary, and J. Litva, IEEE Trans. Microwave Theory Tech. 44, 1115 (1996). 